\title{
Food Additives
}

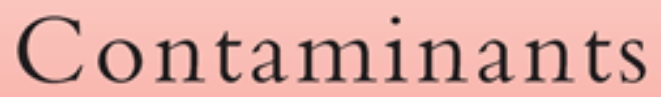

PART A:

CHEMISTRY • ANALYSIS • CONTROL • EXPOSURE \& RISK ASSESSMEN

\section{Multivariate image analysis for the rapid detection of residues from packaging remnants in former foodstuff products (FFPs) - a feasibility study}

\begin{tabular}{|r|l|}
\hline Journal: & Food Additives and Contaminants \\
\hline Manuscript ID & TFAC-2019-499.R1 \\
\hline Manuscript Type: & Original Article \\
\hline Date Submitted by the \\
Author: & $\mathrm{n} / \mathrm{a}$ \\
\hline Complete List of Authors: & $\begin{array}{l}\text { Calvini, Rosalba; University of Modena and Reggio Emilia, Department of } \\
\text { Life Sciences and Interdepartmental Centre BIOGEST-SITEIA } \\
\text { Luciano, Alice; University of Milan, Department of Health, Animal Science } \\
\text { and Food Safety (VESPA) } \\
\text { Ottoboni, Matteo; University of Milan, Department of Health, Animal } \\
\text { Science and Food Safety (VESPA) } \\
\text { Ulrici, Alessandro; University of Modena and Reggio Emilia, Department } \\
\text { of Life Sciences and Interdepartmental Centre BIOGEST-SITEIA } \\
\text { Tretola, Marco; University of Milan, Department of Health, Animal } \\
\text { Science and Food Safety (VESPA); Agroscope, Institute for Livestock } \\
\text { Sciences }\end{array}$ \\
\hline
\end{tabular}


Pinotti, Luciano; University of Milan, Department of Health, Animal Science and Food Safety (VESPA)

Screening assays, Chemometrics

Packaging

Animal feedingstuffs, Animal feed

From a circular economy perspective, feeding livestock with food leftovers or former foodstuff products (FFPs) could be an effective option aimed at exploiting food leftover resources and reducing food losses. FFPs are valuable energy sources, characterized by a beneficial starch/sugar content, and also fats. However, besides these nutritional aspects, safety is a key concern given that FFPs are generally derived from packaged food. Packaging materials, such as plastics and paper, are not accepted as a feed ingredient which means that residues should be rigorously avoided. A sensitive and objective detection method is thus essential for an accurate risk evaluation throughout the former food production chain. To this end, former food samples were collected in processing plants of two different European countries and subjected to Abstract: multivariate analysis of red, green, and blue (RGB) microscopic images, in order to evaluate the possible application of this nondestructive technique for the rapid detection of residual particles from packaging materials. Multivariate Image Analysis (MIA) was performed on single images at the pixel level, which essentially consisted in an exploratory analysis of the image data by means of Principal Component Analysis, which highlighted the differences between packaging and foodstuff particles, based on their colour. The whole dataset of images was then analysed by means of a multivariate data dimensionality reduction method known as the colourgrams approach, which identified clusters of images sharing similar features and also highlighted outlier images due to the presence of packaging particles. The results obtained in this feasibility study demonstrated that MIA is a promising tool for a rapid automated method for detecting particles of packaging materials in FFPs.

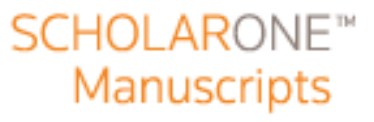




\section{Multivariate image analysis for the rapid detection of residues from packaging}

2 remnants in former foodstuff products (FFPs) - a feasibility study

3 Rosalba Calvini ${ }^{1}$, Alice Luciano ${ }^{2}$, Matteo Ottoboni² ${ }^{2}$, Alessandro Ulrici*1, Marco

4 Tretola ${ }^{2, s}$, Luciano Pinotti ${ }^{2}$

5

$6{ }^{1}$ Department of Life Sciences and Interdepartmental Centre BIOGEST-SITEIA, University of

7 Modena and Reggio Emilia, Pad. Besta, Via Amendola, 2, Reggio Emilia, 42122, Italy

$8{ }^{2}$ Department of Health, Animal Science and Food Safety, VESPA, University of Milan, Milano,

$9 \quad$ Italy

10 \$Present address: Agroscope, Institute for Livestock Sciences, Posieux, Switzerland;

11 * Corresponding author: Prof. Alessandro Ulrici

12 Department of Life Sciences and Interdepartmental Centre BIOGEST-SITEIA, University of

13 Modena and Reggio Emilia, Pad. Besta, Via Amendola, 2, Reggio Emilia, 42122, Italy

14 e-mail: alessandro.ulrici@unimore.it 


\title{
Multivariate image analysis for the rapid detection of residues from packaging remnants in former foodstuff products (FFPs) - a feasibility study
}

\begin{abstract}
From a circular economy perspective, feeding livestock with food leftovers or former foodstuff products (FFPs) could be an effective option aimed at exploiting food leftover resources and reducing food losses. FFPs are valuable energy sources, characterized by a beneficial starch/sugar content, and also fats. However, besides these nutritional aspects, safety is a key concern given that FFPs are generally derived from packaged food. Packaging materials, such as plastics and paper, are not accepted as a feed ingredient which means that residues should be rigorously avoided. A sensitive and objective detection method is thus essential for an accurate risk evaluation throughout the former food production chain. To this end, former food samples were collected in processing plants of two different European countries and subjected to multivariate analysis of red, green, and blue (RGB) microscopic images, in order to evaluate the possible application of this nondestructive technique for the rapid detection of residual particles from packaging materials. Multivariate Image Analysis (MIA) was performed on single images at the pixel level, which essentially consisted in an exploratory analysis of the image data by means of Principal Component Analysis, which highlighted the differences between packaging and foodstuff particles, based on their colour. The whole dataset of images was then analysed by means of a multivariate data dimensionality reduction method known as the colourgrams approach, which identified clusters of images sharing similar features and also highlighted outlier images due to the presence of packaging particles. The results obtained in this feasibility study demonstrated that MIA is a promising tool for a rapid automated method for 37 detecting particles of packaging materials in FFPs.
\end{abstract}




\section{Keywords}

40 Former foodstuffs; packaging remnants; colourgrams; Multivariate Image Analysis (MIA); feed; food 41 safety. 


\section{Introduction}

The livestock sector is inevitably going to involve trade-offs between feed security, feed safety, animal welfare, environmental sustainability and economic development. A common denominator among many of these issues, which are often politically-sensitive, is sustainability. In fact, converting losses from the food industry into ingredients for the feed industry, thereby keeping food losses in the food chain, can be considered a positive cycle that should be implemented worldwide.

Food leftovers or Former Foodstuffs Products (FFPs) are animal feed ingredients consisting of processed and ready-to-eat food products, no longer suitable for human consumption due to logistical, manufacturing or packaging defects. Former food products mainly consist of leftovers from the baking industry (e.g. bread, pasta) and confectionery products (e.g. chocolates, biscuits). Rejected bread, various biscuit products, high-quality baked goods and confectionary from industrial biscuit bakeries are dried and consecutively sorted, unpacked, ground and sieved to create suitable ingredients, which replace some of the existing raw materials in various animal compound feed (Pinotti et al. 2019; Ottoboni et al. 2019; Tretola et al. 2019a, 2019b). Based on the nutritional facts reported for humans, FFPs are extremely rich in carbohydrates, and depending on their origin, also in fats (Giromini et al. 2017). All these factors make FFPs particularly suited to the circular economy: FFPs represent a way by which convert losses from the food industry into ingredients for the feed industry (Pinotti et al. 2019).

Although FFPs are nutritious and safe from a microbiological point of view (Tretola et al. 2017a, 2017b), they may generate other safety issues, such as those related to the presence of packaging remnants. FFPs are un-packaged automatically in order to process a large amount of product. Feed processors routinely remove the packaging from FFPs mechanically in the feed plant; however, despite the removal of most of the packaging, small amounts of wrapping materials remain in the resulting feed. Consequently, a small amount of packaging remnants in the final product (feed) appears to be unavoidable (Tretola et al. 2017a, 2017b). 
Typical remnant residues in FFPs include paper/paperboard, aluminum foil, and plastics, all of which can remain as residues in the final product. In Tretola et al. (2019a) paperboard was the most detected contaminant followed by aluminum foil, and then plastic. Among these, plastics are becoming extremely important especially when small particles are considered. Microplastics are usually defined as plastic particles with a size smaller than $5 \mathrm{~mm}$ for their largest dimension. In general, particles with a size equal to $1-2 \mathrm{~mm}$ or larger can be visually detected, manually extracted and quantified based on weight. This procedure has become a daily practice in the monitoring of former foodstuffs for use in animal feeds (Van Raamsdonk et al. 2020).

However, irrespective of material type, packaging remnants are generally not accepted as a feed ingredient by several authorities, which prohibit the sale of feedstuffs containing packaging materials from the agri-food industry. By contrast, some national authorities have indicated that a minimum percentage of packaging remnants in FFPs is unavoidable and not risky either for animals or humans (Van Raamsdonk et al. 2011; Van Raamsdonk et al. 2012). From a safety point of view, in most analysed samples, the presence of these foreign materials is negligible and below the maximum limit established by some control authorities/bodies (e.g. $0.12 \%$ w/w set by the German Federal Ministry of Food, Agriculture and Consumer Protection) (Pinotti et al. 2019; Tretola et al. 2017a; Tretola et al. 2019a).

In terms of particles dimension, it has been established that remnants normally present in FFPs are mainly in the $>800 \mu \mathrm{m}$ mesh fraction (Tretola et al., 2017a; van Raamsdonk et al., 2012). Quantification of particles smaller than $400 \mu \mathrm{m}$ is generally too laborious and, according to van 87 Raamsdonk et al. (2012), these smaller particles are excluded from the quantification, since their share in the total weight is insignificant.

89 A sensitive and objective detection method is therefore essential for tracing and quantifying 90 packaging remnants in FFPs.

The detection and quantification of packaging remnants in bakery products using a stereomicroscope was proposed by the RIKILT Institute (Wageningen) (Van Raamsdonk et al. 2011; 
Van Raamsdonk et al. 2012). Amato et al. (2017) used a similar approach to develop a sensitive gravimetric method, for routine official controls for the determination of packaging residues in feed.

The two proposed methods can be summarised as follows: (1) visual selection of the undesired ingredients i.e. remnants of packaging materials; (2) weighing of the selected materials; (3) defatting; (4) dehydration; (5) final weighing; and (6) reporting of weight and percentage. In both cases however the methods appear complex, time consuming and analyst sensitive.

In this respect, some of the authors of the present work (Tretola et al. 2017b) used computer sensing to visualize packaging remnants. The results showed that computer vision, when coupled with a stereomicroscope for image acquisition, acts a rapid qualitative screening approach to estimate the presence of foreign materials in food and feed, with little laborious and subjective human visual involvement (Tretola et al. 2017b). Tretola et al. (2019a) also investigated the use of an electronic nose (e-Nose) to detect these contaminants in FFPs. The results indicated that an e-Nose can be used for rapidly screening for the presence of presumed packaging remnants of aluminum, plastics and paperboard in FFPs, when the food/feed matrix is characterized by low variability (e.g. same producer, same odor print).

The aim of this work was to assess the potential of Multivariate Image Analysis (MIA) to automatically detect packaging residues using red, green, and blue (RGB) images of FFP samples acquired with a stereomicroscope. The most common statistical tool applied in MIA is Principal Component Analysis (PCA), which highlights similarities and differences among groups of pixels based on their spectral features (i.e., on their colour for RGB images) (Esbensen et al. 2011; PratsMontalbán et al. 2011; Geladi et al. 1989).

In practical applications for quality monitoring, a high number of images need to be acquired in order to calculate robust and reliable models. In this case, it is necessary to consider both within-image and between-images variability, to properly characterize each single sample and to account at the same time for the variations between the different samples (Gowen et al. 2011; Duchesne et al. 2016; Dorrepaal et al. 2016). Therefore, in order to overcome data handling issues, an image-level approach 
119 is fundamental, which is based on extracting from each image a feature vector summarizing the 120 information needed for the analysis (Ferrari et al. 2013; Calvini et al. 2016).

121 The colourgrams method follows this image-level approach and is specifically implemented for the

0122 11 analysis of RGB images (Antonelli et al. 2004). It converts each RGB image of the dataset into a onedimensional signal, the colourgram, which summarizes the colour features of the corresponding image. The colourgrams are then collected into a data matrix in which each row corresponds to the signal derived from a specific image of the dataset. The colourgrams matrix can then be analysed using common multivariate statistical methods, e.g. PCA, in order to gain an exploratory overview of the whole dataset of images, to identify clusters of images sharing similar features, and to highlight possible outliers.

The colourgrams approach has been successfully applied in several case studies above all related to the analysis of food matrices by means of RGB imaging (Ulrici et al. 2012; Orlandi et al. 2018a; Orlandi et al. 2018b; Giraudo et al. 2018).

In the present study, the images of FFP samples were analysed using both the pixel-level and the image-level approaches, in order evaluate their effectiveness in detecting the presence of possible particles derived from packaging residues.

\section{Materials and methods}

\subsection{FFPs samples and image acquisition}

Six different commercial samples of FFPs, originating from two European countries, were used (Table 1). Three samples (FFPs A, B and D) were obtained from an FFP processing plant in Country 1, while 3 samples (FFPs C, E, F) were from an FFP processor in Country 2.

All samples were produced from different food materials, including bakery products, broken biscuits and chocolates, confectionery products (e.g. croissants, chocolate), surplus bread, rice cakes, salty snacks, and breakfast cereals. For all the FFP samples, a randomly selected aliquot of $5 \mathrm{~g}$ was placed 
in a large Petri dish (PS Ø 90, Colaver, Milan) in a manner to form a single layer. The amount of former food aliquots to be analysed was chosen based on a previous study, which verified homogenous distribution of packaging remnants in reduced amount of former food samples (Tretola et al., 2017a). Specifically, correspondence between packaging remnants levels found in $100 \mathrm{~g}$ of an FFP sample and relative sub-samples of $2 \mathrm{~g}$ was verified by Tretola and coworkers (2017a). In order to increase sampling representativeness, in this study sample quantity was increased to $5 \mathrm{~g}$.

Using a stereo microscope (OLYMPUS SZX9), each sample was investigated separately, taking utmost care in order to avoid any contamination in line with laboratory Standard Operating Procedures (SOPs) for remnants of packaging materials. For each sample, from 5 to 13 images, with or without packaging remnants, were acquired using a digital camera (CoolSNAP-Pro cf Color or AxioCam MRc coupled with a 0.63 port) equipped with an image analysis software (Image-Pro Plus 7.0; Media Cybernetics Inc., Rockville, MD, USA). The pixel size of the acquired RGB images was equal to $1392 \times 1040$, corresponding to a surface area of $2.8 \times 2.1 \mathrm{~mm}$. Therefore, the size of a single pixel was equal to $2 \times 2 \mu \mathrm{m}$, which is much smaller than the minimum size of $400 \mu \mathrm{m}$, as reported by van Raamsdonk et al. (2012).

\subsection{Image analysis}

In order to highlight the potential of MIA to gain a preliminary evaluation at the pixel level of the colour differences between FFP matrices and particles of foreign materials derived from packaging, one image of sample A containing a plastic piece was analysed by means of PCA.

The key aspect of PCA consists in representing a multivariate dataset with a low number of orthogonal variables, named principal components (PCs), which are linear combinations of the original variables (Prats-Montalbán et al. 2011; Geladi et al. 1989). The principal components are calculated so that the first PC (PC1) describes the direction of maximum variance in the data, the second PC (PC2) is orthogonal to $\mathrm{PC} 1$ and accounts for the maximum residual variance (i.e. the variance not described 
by PC1), and the same applies for the subsequent PCs. In order to apply PCA to RGB images, the three-dimensional data array composed of $m$ pixel rows, $n$ pixel columns and the three RGB channels, is unfolded into a bidimensional data matrix with $m \times n$ rows, corresponding to the number of pixels in the image, and three columns corresponding to the RGB values.

The PCA decomposition of the unfolded RGB image (X) can be expressed as follows:

$$
X=T P^{T}+E
$$

where $\mathrm{T}$ is the score matrix containing the pixel coordinates in the PCs space, $\mathrm{P}$ is the loading matrix describing the relevance of the original variables (i.e. the R, G and B channels) in defining the PCs, and $\mathrm{E}$ denotes the residual matrix accounting for residual variation not included in the model.

In order to recover the spatial structure of the image, the score vector of each PC can be refolded into a score image with the same spatial dimensions as the original RGB image.

The same approach described for RGB images can also be applied to more complicated images, such as multispectral or hyperspectral images, which have more than three channels.

In this study, PCA was applied both to the RGB image "as is" as well as to the augmented RGB image that was obtained by considering additional colour-related channels derived from the RGB values.

These additional colour parameters include lightness (L), the relative colours (relative Red, relative Green and relative Blue), and hue (H), saturation (S), and intensity (I) obtained by converting the RGB colour space into the HSI colour space.

Table 2 gives the complete list of the colour-related parameters, together with the corresponding equations.

While in RGB images each pixel is characterized by the three $\mathrm{R}, \mathrm{G}$ and $\mathrm{B}$ channels, in the augmented RGB image, each pixel is defined by seven parameters in addition to the RGB values, for a total of 10 channels. For both images, PCA was applied considering autoscaling as the data preprocessing method.

The whole dataset of 43 images was then analysed at the image-level by converting each image into the corresponding colourgram. The first step in this conversion consists in the same unfolding 
195 procedure described for the pixel-level analysis. The unfolded RGB matrix is then expanded by 196 adding further columns containing additional colour-related parameters. These parameters include 197 the quantities reported in Table 2 and the score vectors obtained by analysing the RGB data matrix 0198 11 12199 13 by means of PCA and considering three preprocessing methods (i.e., no preprocessing, mean centering and autoscaling). For all the variables, the corresponding frequency distribution curves are calculated, considering the entire range of variability of each single variable and dividing it into 256 bins. Then, for each image the corresponding colourgram is calculated by merging in sequence the frequency distribution curves of the considered colour-related parameters and by adding, at the end of the signal, the loading vectors of the PCA models, thereby obtaining a 4900-point long signal. Further details about the algorithm used to calculate the colourgrams can be found in (Antonelli et al. 2004).

The resulting matrix of colourgrams was then analysed by means of PCA using autoscaling as a signal preprocessing method. The exploratory analysis of the dataset at the image-level helped to identify the colour-related features characterizing images of FFP samples with packaging residuals.

The RGB images were converted into the corresponding colourgrams using Colourgrams GUI (Calvini et al., 2020), a user-friendly interface running under MATLAB (The Mathworks, USA). Colourgrams GUI is freely downloadable from http:/www.chimslab.unimore.it/downloads/. The PCA models were calculated using PLS_Toolbox (ver. 8.5, Eigenvector Research Inc., USA) and MIA Toolbox (ver. 3.0.4, Eigenvector Research Inc., USA).

\section{Results}

\subsection{Pixel-level analysis}

To illustrate the potential of MIA performed at the pixel-level, the image reported in Figure 1 was analysed by means of PCA. This image represents a former food matrix contaminated with a semiopaque plastic residue. Figure 1 also shows the corresponding red, green and blue channels, reported separately from each other as gray-scale images. 
221 The PCA model was calculated considering 2 PCs, accounting for $99.76 \%$ of the total variance. In 222 the corresponding PC1-PC2 score plot reported in Figure 2.A, each object represents one pixel of the 223 RGB image. In this plot, the objects are coloured according to the score density, i.e. a yellowish

colour corresponds to an area of the PC1-PC2 score space with a high density of pixels, while blue indicates an area with a low density of pixels. There are two main clusters of pixels in the figure. A comparison of the PC1-PC2 score plot with the corresponding score images reported in Figure 2.CD reveals that the pixels with positive score values for both PC1 and PC2 are mainly ascribable to the piece of plastic. A compact way to simultaneously evaluate the information provided by both PC1 and PC2 is reported in Figure 2.E, which shows the composite false-colour image obtained by superimposing the PC1 and PC2 score images using the red and green channels, respectively.

The PC1 and PC2 loading vectors, reported in Figure 2.B, show which channels contribute most to the separation. The three R, G, and B channels have similar positive loading values on PC1, indicating that the three channels have a comparable contribution in the definition of PC1. Thus, PC1 essentially describes variations of lightness in the image. Conversely, in the PC2 loading vector, the R and B channels have a high influence on the model, while the $\mathrm{G}$ channel has a loading value close to zero. In addition, the $\mathrm{R}$ channel has a negative contribution on $\mathrm{PC} 2$, while the $\mathrm{B}$ channel has a positive contribution on PC2. Therefore, compared to the pixels of the FFP matrix, the pixels of the piece of plastic generally have higher values in the blue channel and lower values in the red channel, while the green channel does not seem to contribute much to separating the two components of the image. In summary, the fact that the plastic residue has high positive scores for both PC1 and PC2, can be ascribed to its brighter and more bluish colour with respect to the FFP.

To better highlight the colour-related differences between the former food and the plastic residue in this image, we also used PCA on the augmented RGB image. The optimal dimensionality of the PCA model was 3 PCs and accounted for $98.92 \%$ of the total variance. In the PC1-PC3 score space reported in Figure 3.A, the pixels of the image are grouped into two main clusters. The pixel cluster lying at positive values of both the PC1 and PC3 score vectors is due to the pixels of the plastic particle. In 
247 fact, the score images of PC1 and PC3 (Figure 3.C and Figure 3.D, respectively) highlight that the 4

pixels of the plastic residue generally have higher scores than the pixels of the FFP. Figure 3.E reports the composite false-colour image of the PC1 and PC3 scores, further confirming the differences between the former food and the plastic residue.

Since the pixels of the plastic particle have high score values for both PC1 and PC3, the colour-related variables that are the most relevant for detecting the plastic fragment are those with loading coefficients of the same sign (i.e. positive or negative) on both PC1 and PC3. For example, relative blue (rB) has positive loading coefficients for both PC1 and PC3, therefore the pixels of the plastic piece have higher $\mathrm{rB}$ values than those of the former food matrix. Conversely, saturation (S) and relative red (rR) have negative loading coefficients on PC1 and PC3, suggesting that the pixels of the plastic residue have lower $\mathrm{S}$ and $\mathrm{rR}$ values than those of the FFP.

In order to confirm the results obtained by PCA, Figure 4.A and Figure 4.B show the gray-scale images of the $\mathrm{rB}$ and $\mathrm{S}$ parameters, respectively. The plastic residue has generally higher $\mathrm{rB}$ values and lower saturation values than the food matrix, as previously found by PCA. The presence of the plastic piece is also much more evident in the $\mathrm{rB}$ and $\mathrm{S}$ gray-scale images than the images of the single RGB channels reported in Figure 1. This suggests that considering additional colour-related parameters better highlights the image features that are not clearly distinguishable from just the R, G and $B$ values.

The histograms of rB and S are reported in Figure 4.C and Figure 4.D, respectively. In both cases, the histograms have a bimodal distribution due to the fact that the plastic fragment and the former food matrix have different $\mathrm{rB}$ and $\mathrm{S}$ values.

In order to verify whether the two peaks in the rB histogram were due above all to the differences between plastic and FFP, a reconstructed image was obtained by visualizing in the original RGB domain only the pixels with $\mathrm{rB}$ values higher than 0.24 (i.e., the pixels whose $\mathrm{rB}$ values fall in the blue area highlighted in Figure 4.C), while the remaining pixels are represented in black. As shown in Figure 4.E, the majority of the selected pixels belong to the plastic residual. The same procedure 
273 was also carried out for the saturation parameter, and in this case only the pixels with S values lower 274 than 0.45 (gray area in Figure 4.D) are reconstructed in Figure 4.F.

\subsection{Image-level analysis}

Concerning the image-level analysis of the whole image dataset, PCA was applied to the colourgram matrix considering $3 \mathrm{PCs}$, which account for $68.67 \%$ of the total variability. In the PC1-PC2 score plot reported in Figure 5.A, each object represents the colourgram of one image, and the objects are coloured according to the corresponding former food sample, while the marker indicates the presence and nature of the packaging residues. For a better interpretation of the results, the labels in the score plot indicate the names of the corresponding images, and the most relevant sample images are also reported.

PC1 separates the images based on the former food type, from the images of sample E to the images of samples A and B. In fact, the former food matrices of sample E have a darker colour, while the former food matrices of samples A and B have a lighter colour.

The trend observed along PC2 suggests that this principal component differentiates between the images according to the presence of white packaging residues, which are primarily due to paper and plastic. In fact, the images with higher PC2 score values included white packaging residues with higher dimensions.

As shown in the PC2-PC3 score plot in Figure 5.B, one image of sample F shows a particular behaviour along PC3, with a much higher score than the other images. In fact, this image contains an aluminium residual with a blue spot, which is not present in the other images with aluminium particles. Figure 5.C reports the Hotelling $\mathrm{T}^{2}$ values and Q residuals of the PCA model. The Hotelling $\mathrm{T}^{2}$ values measure the distance of the samples from the centre of the model (i.e. the origin of the PC space), and therefore describe the variation of each sample within the model. Conversely, the Q residual values indicate how much the description of each sample by the PCA model differs from its actual values. 
298 In other words, samples with high Q residual values show anomalous features, which are not 299 accounted for by the PCA model.

300 Figure 5.C highlights three outlier images: Image 3 has a higher Hotelling $\mathrm{T}^{2}$ value than the corresponding 99.7\% confidence limit, while Image 50 and Image 32 have Q residuals exceeding the $99.7 \%$ confidence limit.

Image 32 has the highest Q residual value, while the corresponding Hotelling $\mathrm{T}^{2}$ value falls within the $95 \%$ confidence limit, thus suggesting that this image has particular features that were not described by the PCA model. In fact, this image shows a red plastic packaging residue, while all the other images with plastic pieces show white or semi-opaque particles.

In order to evaluate the colour features with the greatest influence on the Q residual value of Image 32, the corresponding contribution plot is shown in Figure 6.A. The colourgram regions with the highest contributions are related to the relative green, hue and PC2 and PC3 score vectors of the PCA models calculated with the various preprocessing methods. As in Figure 4, these colour features can be visualized in the original image domain. For example, considering the rG parameter, the peak in the Q contribution plot falls within the 1341-1358 colourgram interval (highlighted in green in Figure 6.A), which corresponds to $\mathrm{rG}$ values ranging from 0.24 and 0.30 . Figure 6.B reports Image 32 in the original RGB colour domain, while Figure 6.C shows the same sample image in which only the pixels falling in the rG interval previously selected are displayed and the remaining pixels are represented in white. The reconstructed pixels are mainly related to the red plastic piece, confirming that the high $\mathrm{Q}$ value of this image is due to the presence of the red packaging residual.

\section{Discussion}

We investigated the potential of RGB imaging coupled with multivariate image analysis strategies for detecting packaging remnants in FFPs. Multivariate image analysis was conducted considering two different approaches: pixel-level analysis and image-level analysis. 
323 The pixel-level approach (Esbensen et al. 2011; Prats-Montalbán et al. 2011) mainly focused on 324 characterizing the individual pixels in an image and in grouping pixels that share similar features. 325 This approach was tested considering both simple RGB images and augmented RGB images obtained by including additional colour-related parameters in the analysis. The results suggest that including additional colour features in the analysis better highlights the differences that are not clearly visible considering the RGB values alone, in particular when the objects that need to be separated have similar colours.

The image-level approach simultaneously compares all the images of the dataset, allowing from tens up to hundreds of RGB images to be analysed together (Antonelli et al. 2004; Ulrici et al. 2012; Orlandi et al. 2018a; Orlandi et al. 2018b; Giraudo et al. 2018). The conversion of the RGB images into colourgrams (Antonelli et al., 2004), highlighted both groups of images with similar colourrelated features and outlier images, e.g. those containing packaging particles. The PCA model calculated on the colourgram matrix showed that the first source of variability in the image dataset was related to the different colour of the former food matrices from different samples. This suggests that in practical scenarios, the development of specific models for each FFP type may lead to more accurate and reliable results. In this case, it will be necessary to acquire and analyse an adequate number of samples representative of each former food type.

PCA also highlighted common trends in images with white residues derived from paper or plastic packaging materials and to identify images showing particular features due to the presence of aluminium or differently-coloured plastic remnants.

Outlier images can be easily detected considering the Hotelling $\mathrm{T}^{2}$ values and Q residuals, which can be used to build multivariate control charts or classification models capable of automatically detecting images with packaging remnants.

However, RGB imaging only accounts for colour properties of the imaged samples, which is one limitation of this technique in the detection of packaging materials in FFPs. In fact, in some cases, the packaging residues may be too similar in colour to the FFP particles, making them difficult to 
identify (Tretola et al., 2017a; Tretola et al., 2017b). For example, paperboard is particularly difficult to differentiate from feed material, making its detection complicated and time consuming.

In order to overcome this issue, the stereomicroscope could be coupled with more advanced imaging systems capable of detecting light also beyond the visible spectral region, including for example the near infrared spectral region (Gowen et al., 2011; Ferrari et al., 2013; Dale et al., 2013; Ulrici et al., 2013; Amigo et al., 2015; Calvini et al., 2016; Vermeulen et al., 2017; Calvini et al., 2018). The colour features of the samples derived from RGB images could be combined with spectral features derived from multispectral or hyperspectral images, thus leading to a more comprehensive characterization of the differences between former food matrices and residues of packaging materials.

\section{Conclusions}

The present work is a preliminary study focused on the potential of imaging methods applied to feed and food safety. Identifying packaging remnants in former food products is important in ensuring the safety of FFPs used as feed ingredients. Generally, control procedures for the determination of packaging residues in feed are based on the visual inspection and manual selection of undesirable contaminant materials.

To overcome the drawbacks of these procedures, we have explored the feasibility of using RGB imaging as a rapid and non-destructive tool for the automated detection of packaging particles in FFPs. In this paper, we have assessed the various features of this technique, together with the challenges related to the application of image analysis strategies.

The preliminary results obtained in this study demonstrate the potential of the proposed approach, in particular when the colour of the undesirable packaging residues can be differentiated from the colour of the ex-food matrix.

In order to develop more robust and sensitive models, we plan to increase the size of the image dataset by acquiring a higher number of images representative of each FFP type. 


\section{6. Acknowledgements}

376 The present work has been done in the frame of the following projects: Sustainable feed design 377 applying circular economy principles: the case former food in pig nutrition (SUSFEED) funded by 10378 the Fondazione Cariplo, call "Economia circolare: ricerca per un futuro sostenibile"; Progetto di

379 Grande Rilavenza IT-RS "Sustainable animal nutrition" (SUN) funded the Ministero degli Affari 14 15380 16

\section{Declaration of interest statement}

Esteri e della Cooperazione Internazionale.

\section{The authors declare that they have no conflict of interest.}




\section{References}

Amato G, Desiato R, Giovannini T, Pinotti L, Tretola M, Gili M, Marchis D. 2017. Gravimetric quantitative determination of packaging residues in feed from former food. Food Addit Contam Part A. 34(8), 1446-1450. doi: 10.1080/19440049.2017.1337277

Amigo JM, Babamoradi H, Elcoroaristizabal S. 2015. Hyperspectral image analysis. A tutorial. Analytica Chimica Acta. 896, 34-51. doi: 10.1016/j.aca.2015.09.030

Antonelli A, Cocchi M, Fava P, Foca G, Franchini GC, Manzini D, Ulrici A. 2004. Automated evaluation of food colour by means of multivariate image analysis coupled to a wavelet-based classification algorithm. Analytica Chimica Acta. 515(1), 3-13. doi: 10.1016/j.aca.2004.01.005

Calvini R, Foca G, Ulrici A. 2016. Data dimensionality reduction and data fusion for fast characterization of green coffee samples using hyperspectral sensors. ABC. 408(26), 7351-7366. doi: 10.1007/s00216-016-9713-7

Calvini R, Orlandi G, Foca G, Ulrici A. 2018. Development of a classification algorithm for efficient handling of multiple classes in sorting systems based on hyperspectral imaging. JSI. 7, a13. doi: $10.1255 /$ jsi.2018.a13

Calvini R, Orlandi G, Foga G, Ulrici A. 2020. Colourgrams GUI: A graphical user-friendly interface for the analysis of large datasets of RGB images. Chemom. Intell. Lab. Syst. 196(15), 103915. doi: 10.1016/j.chemolab.2019.103915

Cheli F, Bontempo V, Pinotti L, Ottoboni M, Tretola M, Baldi A, Dell'Orto V. 2018. Feed Analysis and Animal Nutrition: Electronic Nose as a Diagnostic Tool. Chem Eng Trans. 68, 223-228.

Dale LM, Thewis A, Boudry C, Rotar I, Dardenne P, Baeten V, Pierna JAF. 2013. Hyperspectral imaging applications in agriculture and agro-food product quality and safety control: A review. Appl Spectrosc Rev. 48(2), 142-159. doi: 10.1080/05704928.2012.705800

Dorrepaal R, Malegori C, Gowen A. 2016. Tutorial: Time series hyperspectral image analysis. J Near Infrared Spec. 24(2), 89-107. doi: 10.1255/jnirs.1208

Duchesne C, Liu JJ, MacGregor JF. 2012. Multivariate image analysis in the process industries: A review. Chemom Intell Lab Syst. 117, 116-128. doi: 10.1016/j.chemolab.2012.04.003

Esbensen K, Geladi P. 1989. Strategy of multivariate image analysis (MIA). Chemom Intell Lab Syst. 7(1-2), 67-86. doi: 10.1016/0169-7439(89)80112-1

Ferrari C, Foca G, Ulrici A. 2013. Handling large datasets of hyperspectral images: Reducing data size without loss of useful information. Analytica Chimica Acta. 802, 29-39. doi: 10.1016/j.aca.2013.10.009

Geladi P, Isaksson H, Lindqvist L, Wold S, Esbensen K. 1989. Principal component analysis of multivariate images. Chemom Intell Lab Syst. 5(3), 209-220. doi: 10.1016/0169-7439(89)80049-8

Giraudo A, Calvini R, Orlandi G, Ulrici A, Geobaldo F, Savorani F. 2018. Development of an automated method for the identification of defective hazelnuts based on RGB image analysis and colourgrams. Food Control. 94, 233-240. doi: 10.1016/j.foodcont.2018.07.018 
Giromini C, Ottoboni M, Tretola M, Marchis D, Gottardo D, Caprarulo V, Baldi A, Pinotti L. 2017. Nutritional evaluation of former food products (ex-food) intended for pig nutrition. Food Add Contam Part A. 34(8), 1436-1445.

Gowen AA, Marini F, Esquerre C, O’donnell C, Downey G, Burger J. 2011. Time series hyperspectral chemical imaging data: challenges, solutions and applications. Analytica Chimica Acta. 705(1-2), 272-282. doi: 10.1016/j.aca.2011.06.031

Kucheryavskiy S. 2013. A new approach for discrimination of objects on hyperspectral images. Chemom Intell Lab Syst. 2013 120, 126-135. doi: 10.1016/j.chemolab.2012.11.009

Orlandi G, Calvini R, Foca G, Ulrici A. 2018a. Automated quantification of defective maize kernels by means of Multivariate Image Analysis. Food Control. 85, 259-268. doi: 10.1016/j.foodcont.2017.10.008

Orlandi G, Calvini R, Pigani L, Foca G, Simone, G. V., Antonelli, A., Ulrici, A. 2018b. Electronic eye for the prediction of parameters related to grape ripening. Talanta. 186, 381-388. doi: 10.1016/j.talanta.2018.04.076

Ottoboni M, Tretola M, Luciano A, Giuberti G, Gallo A, Pinotti L. 2019. Carbohydrate digestion and predicted glycemic index of bakery/confectionary ex-food intended for pig nutrition. Italian Journal of Animal Science. 1, 838-849. doi: 10.1080/1828051X.2019.1596758

Pinotti L, Giromini C, Ottoboni M, Tretola M, Marchis D. 2019. Review: insects and former foodstuffs for upgrading food waste biomasses/streams to feed ingredients for farm animals. Animal. 1-11. doi: $10.1017 / \mathrm{S} 1751731118003622$

Prats-Montalbán, J. M., De Juan, A., Ferrer, A. 2011. Multivariate image analysis: a review with applications. Chemometrics and Intelligent Laboratory Systems. 107(1), 1-23. doi: 10.1016/j.chemolab.2011.03.002

Tretola M, Di Rosa A, Tirloni E, Ottoboni M, Giromini C, Leone F, Bernardi CEM, Dell'Orto V, Chiofalo V, Pinotti L. 2017a. Former food products safety: microbiological quality and computer vision evaluation of packaging remnants contamination. Food Addit Contam Part A. 34(8), 14271435. doi: 10.1080/19440049.2017.1325012

Tretola M, Ottoboni M, Di Rosa A, Giromini C, Fusi E, Rebucci R, Leone F, Dell'Orto V, Chiofalo V, Pinotti L. 2017 b. Former Food Products Safety Evaluation: Computer Vision as an Innovative Approach for the Packaging Remnants Detection. J Food Qual. 1-6. doi: 10.1155/2017/1064580

Tretola M, Ottoboni M, Luciano A, Dell'Orto V, Cheli F, Pinotti L. 2019a.Tracing food packaging contamination: an electronic nose applied to leftover food. Food Addit Contam Part A. 36, 1748-1756. doi: $10.1080 / 19440049.2019 .1653498$

Tretola M, Ottoboni M, Luciano A, Rossi L, Baldi A, Pinotti L. 2019b. Former food products have no detrimental effects on diet digestibility, growth performance and selected plasma variables in postweaning piglets. IJAS. 18, 987-996. doi.org/10.1080/1828051X.2019.1607784

Tretola M, Luciano A, Ottoboni M, Baldi A, Pinotti L. 2019c. Influence of Traditional vs Alternative Dietary Carbohydrates Sources on the Large Intestinal Microbiota in Post-Weaning Piglets. Animals. 9(8), 516. https://doi.org/10.3390/ani9080516. 
462 Ulrici A, Foca G, Ielo MC, Volpelli LA, Lo Fiego DP. 2012. Automated identification and 463 visualization of food defects using RGB imaging: Application to the detection of red skin defect of 464 raw hams. Innov Food Sci Emerg Tech. 16, 417-426. doi: 10.1016/j.ifset.2012.09.008

465 Ulrici A, Serranti S, Ferrari C, Cesare D, Foca G, Bonifazi G. 2013. Efficient chemometric strategies 466 for PET-PLA discrimination in recycling plants using hyperspectral imaging. Chemom Intel Lab Syst. 10467 11

van Raamsdonk LWD, Pinckaers VGZ., Vliege JJM, van Egmond HJ. 2012. Examination of packaging materials in bakery products: a validated method for detection and quantification. RIKILT Report - Wageningen, the Netherlands. 1-20.

van Raamsdonk LWD, Rijk R, Schouten GPJ, Mennes W, Meijer GAL, Poel AFBVD, De Jong J. 2011. A risk evaluation of traces of packaging materials in former food products intended as feed materials. RIKILT Report - Wageningen, the Netherlands. 1-69.

van Raamsdonk LWD, van der Zande M, Koelmans AA, Hoogenboom RL, Peters RJ, Groot MJ, Peijnenburg Ad ACM, Weesepoel, Y. J. 2020. Current Insights into Monitoring, Bioaccumulation, and Potential Health Effects of Microplastics Present in the Food Chain. Foods, 9(1), 1-28. doi:10.3390/foods 9010072

Vermeulen P, Ebene MB, Orlando B, Fernández Pierna JA, Baeten V. 2017. Online detection and quantification of particles of ergot bodies in cereal flour using near-infrared hyperspectral imaging. Food Add Contam Part A. 34(8), 1312-1319. doi: 10.1080/19440049.2017.1336798 

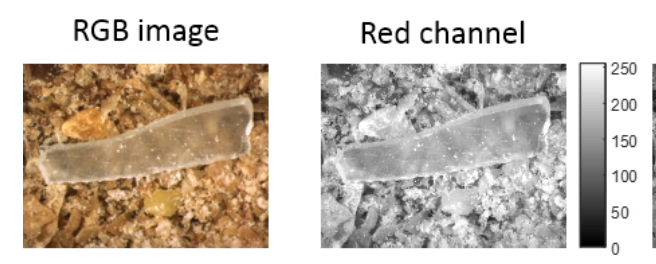

Green channel

Blue channel

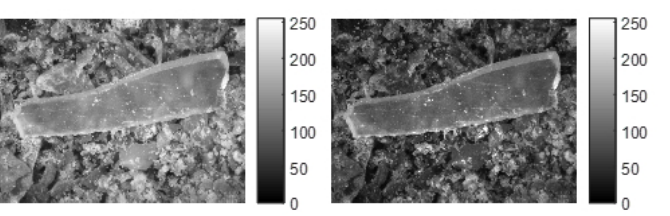

Figure 1. RGB image of a sample contaminated with a plastic residue and gray-scale images of the corresponding red, green and blue channels.

$256 \times 71 \mathrm{~mm}(96 \times 96 \mathrm{DPI})$ 

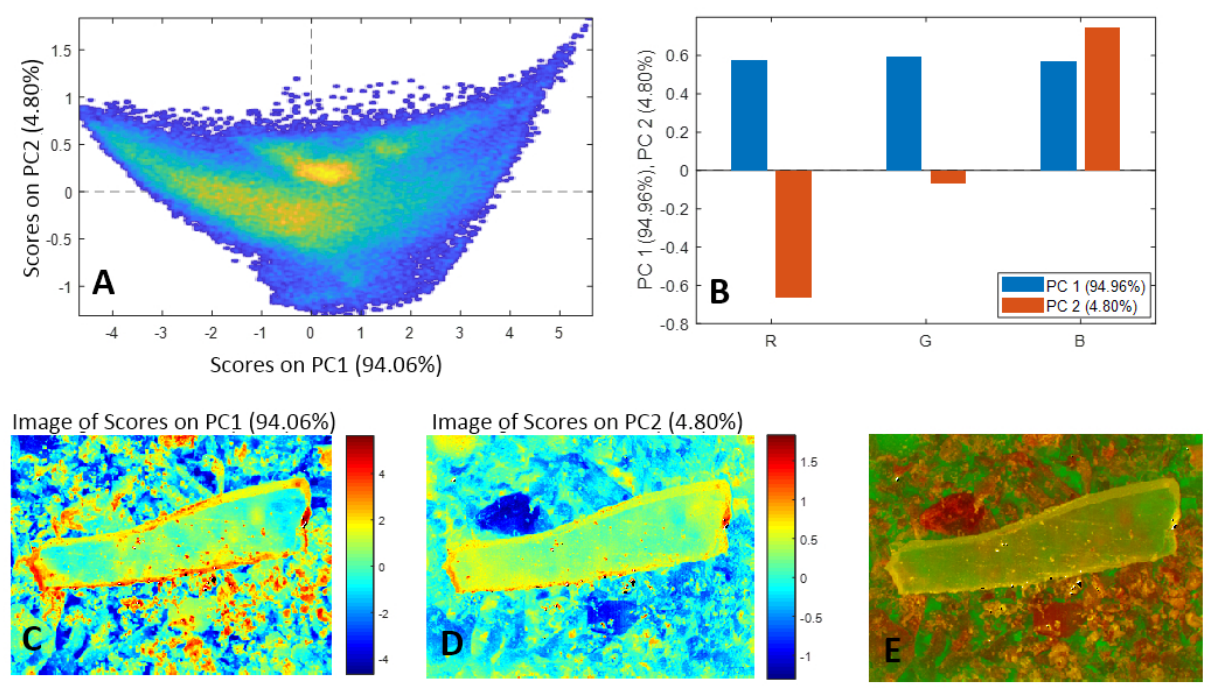

Figure 2. Results of the PCA model calculated on the RGB image of a former food sample with a plastic residue. In A: PC1 - PC2 score plot; in B: PC1 - PC2 loading plot; in C: PC1 score image; in D: PC2 score image and in E: false-colour PC1-PC2 score image.

\section{$295 \times 166 \mathrm{~mm}(96 \times 96 \mathrm{DPI})$}



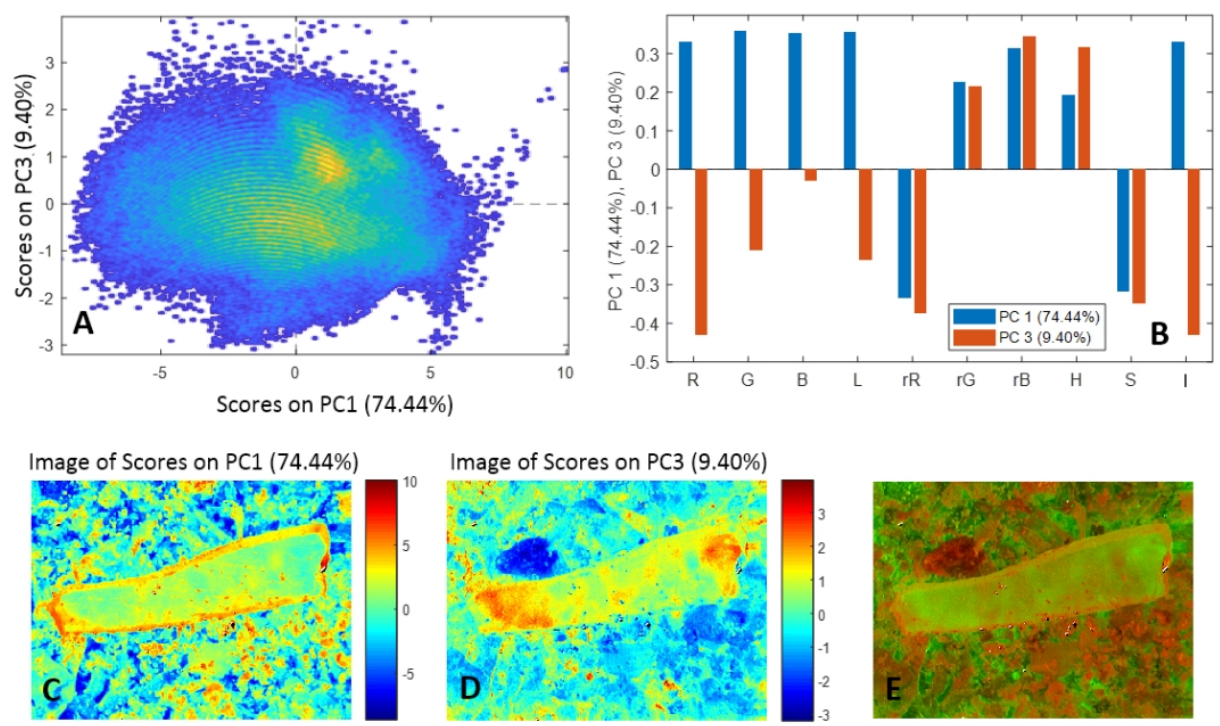

Figure 3. Results of the PCA model calculated on the augmented RGB image of a former food sample with a plastic residue. In A: PC1 - PC3 score plot; in B: PC1 - PC3 loading plot; in C: PC1 score image; in D: PC3 score image and in E: false-colour PC1-PC3 score image.

$317 \times 189 \mathrm{~mm}(96 \times 96 \mathrm{DPI})$ 

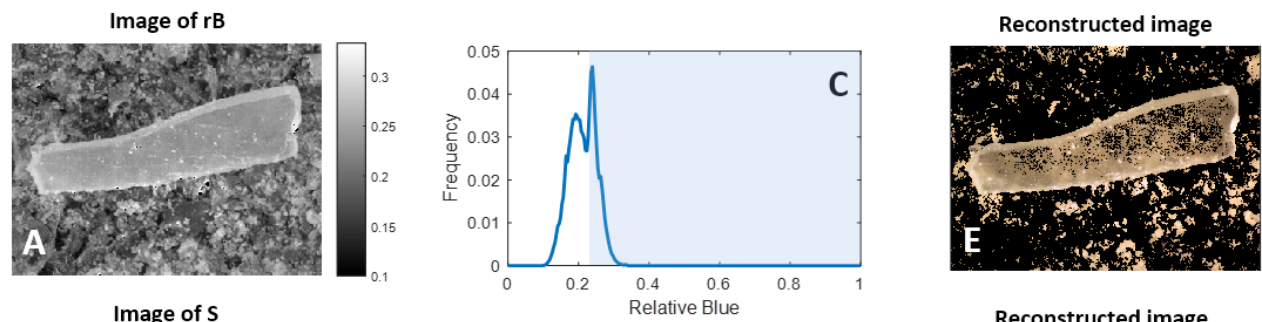

Image of S
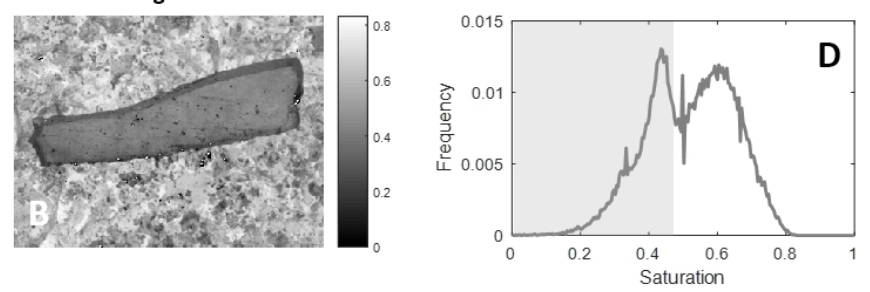

Reconstructed image

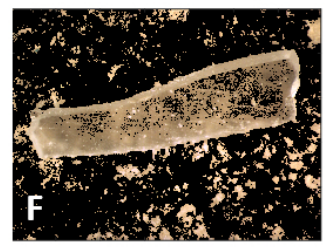

Figure 4. Gray-scale images of relative blue $(A)$ and saturation (B); frequency distribution curves of relative blue (C) and saturation (D); images reconstructed using the selected features of relative blue (E) and saturation $(F)$.

$293 \times 150 \mathrm{~mm}(96 \times 96 \mathrm{DPI})$ 

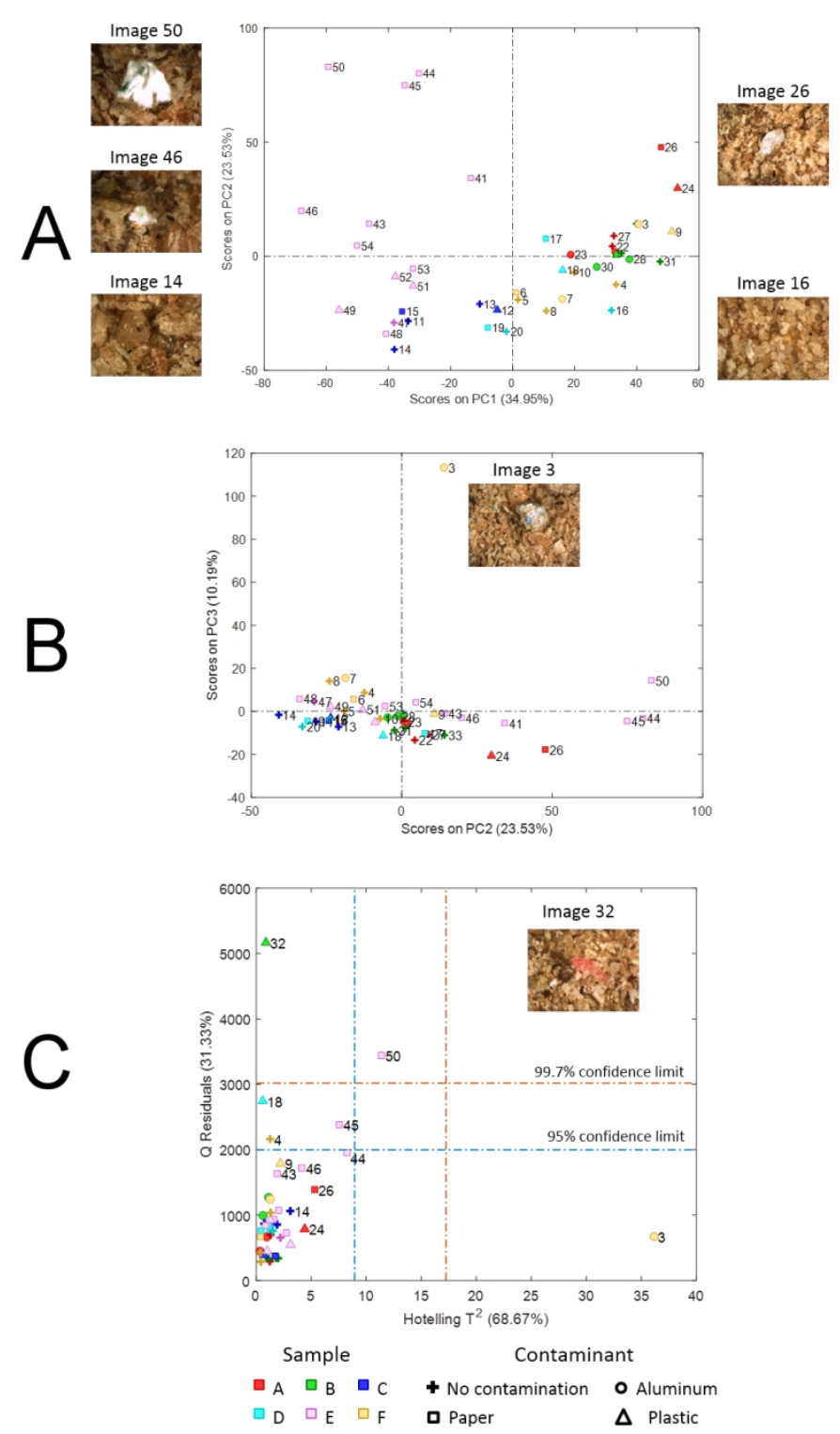

Results of the PCA model calculated on the colourgrams matrix. In A: PC1-PC2 score plot; in B: PC2-PC3 score plot; in C: Hotelling T2 and Q residuals plot.

$89 \times 159 \mathrm{~mm}(300 \times 300 \mathrm{DPI})$ 

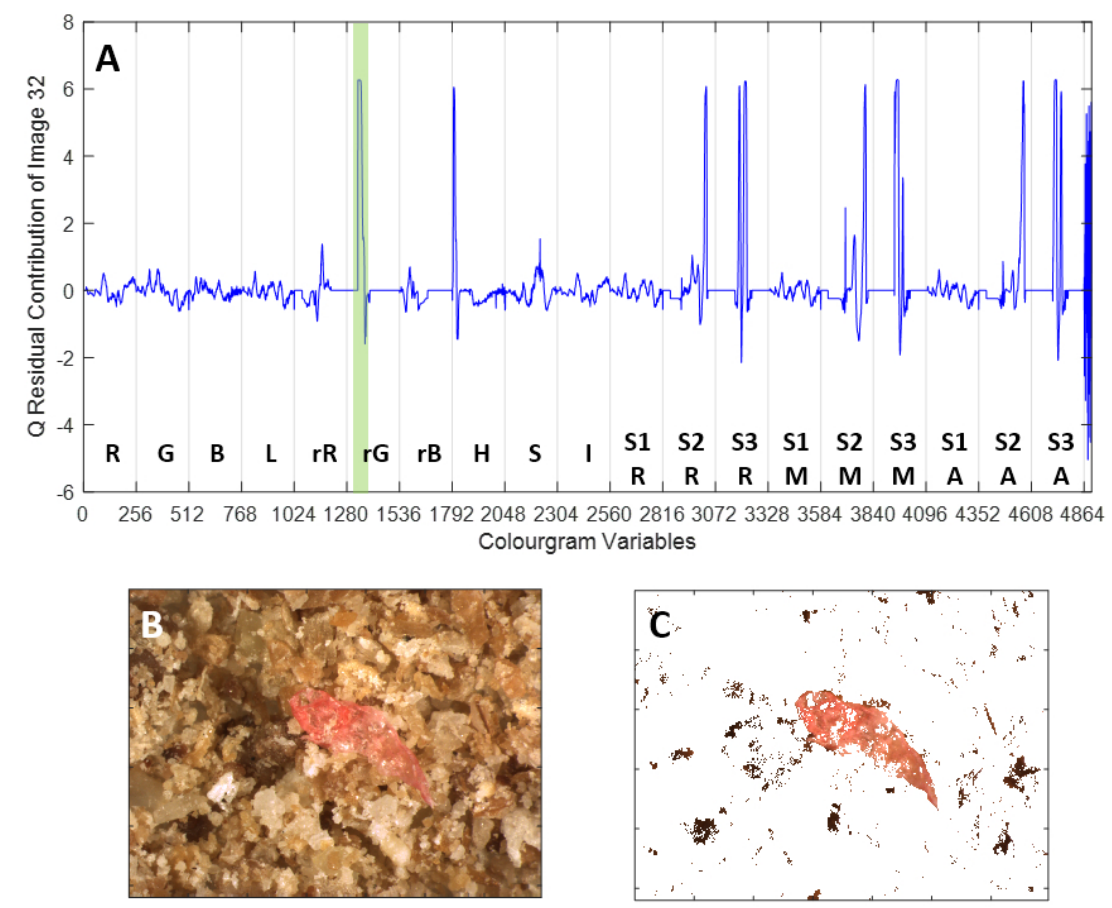

$Q$ residual contribution plot of Image $32(A)$, original Image 32(B), and Image 32 reconstructed using the selected features $(C)$.

$87 \times 62 \mathrm{~mm}(300 \times 300$ DPI $)$ 
Table 1. Summary of the FFP samples considered in this study and corresponding number of acquired images.

\begin{tabular}{|c|c|c|c|c|c|c|}
\hline $\begin{array}{c}\text { Sample } \\
\text { name }\end{array}$ & $\begin{array}{c}\text { Total n } \\
\text { of images }\end{array}$ & $\begin{array}{c}\text { Main food } \\
\text { product } \\
\text { types }\end{array}$ & $\begin{array}{c}\text { Images } \\
\text { with no } \\
\text { residues }\end{array}$ & $\begin{array}{c}\text { Images } \\
\text { with plastic } \\
\text { residues }\end{array}$ & $\begin{array}{c}\text { Images } \\
\text { with paper } \\
\text { residues }\end{array}$ & $\begin{array}{c}\text { Images with } \\
\text { aluminum } \\
\text { residues }\end{array}$ \\
\hline $\mathbf{A}$ & 6 & $\begin{array}{c}\text { confectionery } \\
\text { products }\end{array}$ & 2 & 1 & 1 & 2 \\
\hline $\mathbf{B}$ & 6 & $\begin{array}{c}\text { confectionery } \\
\text { products }\end{array}$ & 3 & 1 & 0 & 2 \\
\hline $\mathbf{C}$ & 5 & $\begin{array}{c}\text { confectionery } \\
\text { products }\end{array}$ & 3 & 1 & 1 & 0 \\
\hline $\mathbf{D}$ & 5 & $\begin{array}{c}\text { baked goods } \\
\text { and } \\
\text { confectionery } \\
\text { products }\end{array}$ & 2 & 1 & 2 & 0 \\
\hline $\mathbf{E}$ & 13 & $\begin{array}{c}\text { confectionery } \\
\text { products }\end{array}$ & 1 & 3 & 9 & 0 \\
\hline $\mathbf{F}$ & 8 & $\begin{array}{c}\text { baking } \\
\text { industry }\end{array}$ & 4 & 1 & 1 & 2 \\
\hline Total & $\mathbf{4 3}$ & $\mathbf{1 5}$ & $\mathbf{8}$ & $\mathbf{1 4}$ & $\mathbf{6}$ \\
\hline
\end{tabular}


Table 2. Colour-related parameters used to calculate the augmented RGB image.

\begin{tabular}{|c|c|c|}
\hline Name & Abbreviation & Equation \\
\hline Lightness & $\mathrm{L}$ & $\mathrm{L}=\mathrm{R}+\mathrm{G}+\mathrm{B}$ \\
\hline Relative Red & $\mathrm{rR}$ & $\mathrm{rR}=\mathrm{R} / \mathrm{L}$ \\
\hline Relative Green & $\mathrm{rG}$ & $\mathrm{rG}=\mathrm{G} / \mathrm{L}$ \\
\hline Relative Blue & $\mathrm{rB}$ & $\mathrm{rB}=\mathrm{B} / \mathrm{L}$ \\
\hline Hue & $\mathrm{H}$ & $\begin{array}{l}\text { Value ranging from 0 to 1, corresponding to a colour } \\
\text { transition from red to orange, yellow, green, cyan, blue, } \\
\text { magenta, and finally back to red. }\end{array}$ \\
\hline Saturation & $\mathrm{S}$ & $\mathrm{S}=[\max (\mathrm{R}, \mathrm{G}, \mathrm{B})-\min (\mathrm{R}, \mathrm{G}, \mathrm{B})]$ / max $(\mathrm{R}, \mathrm{G}, \mathrm{B})$ \\
\hline Intensity & $\mathrm{I}$ & $\mathrm{I}=\max (\mathrm{R}, \mathrm{G}, \mathrm{B})$ \\
\hline
\end{tabular}

